\title{
Orbital decay of satellites crossing an accretion disc
}

\author{
V. Karas and L. Šubr \\ Astronomical Institute, Charles University Prague, V Holešovičkách 2, 18000 Praha, Czech Republic \\ e-mail: vladimir.karas@mff.cuni.cz; subr@aglaja.ms.mff.cuni.cz \\ Received 16 January 2001 / Accepted 8 June 2001

\begin{abstract}
Motion of stellar-mass satellites is studied around a massive compact body which is surrounded by a gaseous slab of a stationary accretion disc. The satellites suffer an orbital decay due to hydrodynamical interaction with the disc medium (transitions across the disc, gap opening in the disc, density waves) and gravitational radiation. Arbitrary orbital eccentricities and inclinations are considered, and it is observed how the competing effects depend on the parameters of the model, namely, the mass and compactness of the orbiters, the osculating elements of their trajectories, and surface density of the disc. These effects have a visible impact on the satellites long-term motion, and they can produce observational consequences with respect to galactic central clusters. It is shown that the satellite-disc collisions do not impose serious restrictions on the results of gravitational wave experiments if the disc medium is diluted and the orbiter is compact but they are important in the case of environments with relatively high density. We thus concentrate on application to accretion flows in which the density is not negligible. We discuss the expected quasi-stationary structure of the cluster that is established on sub-parsec scales within the sphere of gravitational influence of the central object. Relevant to this region, we give the power-law slopes defining the radial profile of modified clusters and we show that their values are determined by satellite interaction with the accretion flow rather than their initial distribution.
\end{abstract}

Key words. accretion, accretion-discs - galaxies: nuclei

\section{Introduction}

There is ample evidence that central regions of galaxies consist of three main constituent parts: a supermassive dark object (presumably a black hole), a dense cluster of stars, and an accretion flow with disc-type geometry (for a review, see Rees 1998; Kato et al. 1998). Related to these three ingredients are typical lengthscales: (i) $r_{\mathrm{g}}=G M / c^{2} \approx 1.5 \times 10^{13} M_{8} \mathrm{~cm}$ (gravitational radius of the central black hole expressed in terms of its mass $M_{8} \equiv M / 10^{8} M_{\odot} \lesssim 1$ ). (ii) $r_{\mathrm{c}}=G M / \sigma^{2} \approx$ $0.5 M_{8} \sigma_{1000}^{2} \mathrm{pc}$, the size of an inner star-cluster (where motion of its members is governed mainly by the black-hole gravitational field) written in terms of stellar velocity dispersion $\sigma_{1000} \equiv \sigma /\left(1000 \mathrm{~km} \mathrm{~s}^{-1}\right)$. A typical number of stars in this region can be of the order $N \approx 10^{5}$. Finally, (iii) $r_{\mathrm{d}} \approx 10^{4} r_{\mathrm{g}}$ (the size of the disc). For the purpose of this paper these stars play a role of stellar-mass satellites orbiting the center. The disc itself will be described in the thin-disc approximation with the total mass $M_{\mathrm{d}} \ll M$.

In this paper we assume that the three mentioned subsystems coexist and they form an integral structure for a sufficiently long period of time. This is a reasonable

Send offprint requests to: V. Karas,

e-mail: vladimir .karas@mff .cuni.cz assumption for a range of model parameters (discussed below), and the resulting geometry captures interesting effects of realistic systems, although it is simplified in several respects. The three subsystems are of very different nature, so that their symbiosis and mutual interactions pose a complex, ecological problem (e.g., Shlosman \& Noguchi 1993; van der Marel \& van den Bosch 1998; Zwart et al. 1997). We confine ourselves to a simple analysis in which the gravitational field of the system is dominated by the central compact mass while long-term dynamics of the satellites is influenced by dissipation in the disc medium and by gravitational radiation. The present paper is motivated mainly by indications of substantial concentrations of both stars and interstellar medium in the galactic central parsec. For example, a toroidal system of stars and gas has been proposed as a plausible explanation of brightness peaks observed in the nucleus of M 31 (Tremaine 1995; Bekki 2000). However, as we want to discuss also the regime in which inspiraling satellites are influenced by gravitational radiation in the field of the massive centre, we need to consider sub-parsec scales which are not yet resolved observationally. This appears relevant for the continuation (towards small length-scales) of the velocity dispersion versus mass relation (Ferrarese \& Merritt 2000), which is so important in the discussion of the masses of 
supermassive black holes. Finally, core-collapsed globular clusters could be another class of relevant objects on scales of masses $\approx 10^{4} M_{\odot}$, i.e. much less than galactic cores.

We proceed along the same course as several recent works in which further references can be found: Rauch (1999) studied stellar dynamics near a massive black hole, including the effects of general relativity on stellar trajectories; Subr \& Karas (1999) examined the long-term evolution of orbital parameters of a satellite colliding with a thin Keplerian disc; Narayan (2000) estimated the relative importance of hydrodynamic drag versus gravitationalradiation decay of the satellite orbit (this author was interested primarily in the case of a body embedded in an advection-dominated flow very near the central hole); Collin \& Zahn (1999), on the other hand, explored the fate of satellites moving on the periphery of the disc and at intermediate distances $\left(\approx 1\right.$ pc for $\left.M_{8} \approx 1\right)$ where mutual interaction of the disc matter with the satellites triggers the starburst phenomenon and provides a mechanism of metal enrichment; finally, Takeuchi et al. (1996) and Ward (1997) discussed the problem of satellite migration via gap formation and density waves in the disc, and they gave a detailed overview of related topics which have been widely discussed also by others.

The orbital motion of satellites forming the central cluster is dominated by gravity of the central body; however, the long-term evolution of their trajectories is affected also by many other effects such as gravity of the background galaxy (e.g., Sridhar \& Touma 1999), dynamical friction and tidal interactions (Colpi et al. 1999), selfgravity of the disc matter (Shlosman \& Begelman 1989; Vokrouhlický \& Karas 1998), repetitive collisions with an accretion disc (Syer et al. 1991; Rauch 1995), and gravitational radiation losses (Peters \& Mathews 1963). The interaction between the members of the cluster and the disc are of interest also because they may gradually change the composition of the disc (Artymowicz et al. 1993), speed up the evolution of the satellite itself (Collin \& Zahn 1999), and they can enrich the environment outside the disc plane (Zurek et al. 1994; Armitage et al. 1996).

Using simple estimates we compare the energy and angular momentum losses via gravitational radiation against the hydrodynamical drag acting on the satellites. We assume that their orbits have, initially, arbitrary eccentricities and nonzero inclinations with respect to the disc plane - the situation which is complementary to the case of planetary formation and their migration inside the disc. For the subsequent (low-inclination) stages of the orbital evolution we employ a simple prescription in which gravitational radiation losses still tend to bring the orbiter towards the centre while hydrodynamical effects are approximated in terms of motion through gas.

We build our discussion on a model description (Šubr \& Karas 1999) in which the satellite suffers from dissipation of the orbital energy during repetitionary star-disc interactions but the disc itself does not change with time. We show evolutionary tracks followed by the satellite in the parameter space of its orbital osculating elements.
The results are found quite sensitive to actual values of the disc density, the mass of the satellite, and to several other parameters of the model. We discuss the dynamical evolution of a cluster of satellites which gradually departs from its initially spherical shape and evolves into a flattened system. The initial stage of the evolution (when most of the orbits are still inclined with respect to the disc) is similar to the situation discussed by Pineault \& Landry (1994) and Rauch (1995), but for the later stages we must adapt the prescription for star-disc interactions. See also Ivanov et al. (1999) for a complementary discussion of the gradual evolution of a circumbinary disc structure.

\section{Satellite motion influenced by the disc}

The satellite body is assumed to cross the disc at supersonic velocity with a high Mach number, $\mathcal{M} \approx 10^{2}-10^{3}$. We recall (Syer et al. 1991; Vokrouhlický \& Karas 1993; Zurek et al. 1994) that passages last a small fraction of the orbital period at the corresponding radius where they occur, and they can be considered as instantaneous, repetitive events at which the passing satellite expels from the disc some amount of material that lies along its trajectory. In terms of inclination angle $i$ and the disc thickness $h$, the typical ratio of the two periods is $\delta \approx h /(r \sin i)$; this quantity is assumed to be less than unity. Hence, for a geometrically thin $\operatorname{disc}(\epsilon \equiv h / r \ll 1)$, its influence upon inclined stellar orbits can be treated as tiny kicks (impulsive changes of their energy and momentum) at the points of intersection with the plane of the disc. This can be translated using the relation for the speed of sound, $c_{\mathrm{s}}=\epsilon \Omega r$, to the claim that the motion across the disc is indeed supersonic, which is also required for consistency. Smallness of $\delta$ means that the satellite remains outside the disc for most of its revolution around the centre; naturally this condition cannot be ensured at final stages when $i$ is very small.

\subsection{Collisions with the disc}

Repetitive collisions lead to the gradual change of the orbiter's velocity $\boldsymbol{v} \rightarrow \boldsymbol{v}^{\prime}$, which can be expressed by momentum conservation (Šubr \& Karas 1999):

$(A+1) \boldsymbol{v}^{\prime}=v_{\mathrm{r}} \boldsymbol{e}_{\boldsymbol{r}}+v_{\vartheta} \boldsymbol{e}_{\vartheta}+\left(v_{\varphi}+A v_{\mathrm{K}}\right) \boldsymbol{e}_{\boldsymbol{\phi}}$

in spherical coordinates $(\vartheta=\pi / 2$ is the disc plane). Here, $A(r) \equiv \Sigma_{\mathrm{d}} v_{\text {rel }} \Sigma_{*}^{-1} v_{\vartheta}^{-1}, v_{\text {rel }}$ is the relative speed between the orbiter and the disc matter, $\Sigma_{*}$ is the column density characterizing the compactness of the orbiter and defined by $\Sigma_{*}=M_{*} /\left(\pi R_{*}^{2}\right)$ (quantities denoted by an asterisk refer to the orbiter, $\left.M_{*} \ll M\right)$, and $\Sigma_{\mathrm{d}}(r)$ is the disc surface density. Rotation of the disc is assumed Keplerian, $v_{\phi} \equiv v_{\mathrm{K}}=\sqrt{G M / r}$.

Let us consider a satellite on an orbit with semi-major axis $a$, eccentricity $e$, inclination $i$, and longitude $\omega$ of the ascending node. Equation (1) implies a set of equations which can be solved numerically in terms of the orbiter's osculating elements, while analytical solutions are possible 
in special cases (Šubr \& Karas 1999). As a useful example, we assume a power-law surface-density distribution in the form $\Sigma_{\mathrm{d}}=K\left(r / r_{\mathrm{g}}\right)^{s} \Sigma_{\odot}(K=$ const. $)$ and we adopt a perpendicular orientation of the orbit, $\cos \omega=0$. We find

$a=C_{1} x_{+}\left(x_{+}^{3}+C_{2} x_{-}\right)^{-1}, \quad y=1+C_{2} x_{-} x_{+}^{-3}$,

where $x_{ \pm} \equiv 1 \pm x, x \equiv \cos i, y \equiv 1-e^{2}$. Strictly speaking, this formula concerns only the case of orbits intersecting the disc at two points with identical radial distances from the center but it can be used also as an approximation for orbits with arbitrary orientation. We remark that the relative accuracy $\Delta a / a$ of the determination of the semimajor axis is better than $15 \%$ with reasonable density profiles ( $s$ of the order of unity); numerical computations are not limited by assumptions about $\omega$ imposed in Eq. (2). Furthermore, one can write

$A=\frac{K \Sigma_{\odot}}{\Sigma_{*}}\left(\frac{a y}{r_{\mathrm{g}}}\right)^{s} \sqrt{\frac{3-y-2 x}{1-x^{2}}}$.

Integration constants $C_{1,2}$ are to be determined from initial values of $a=a_{0}, x=x_{0}$, and $y=y_{0}$. Then, the temporal history is obtained by integrating over the orbital period, $\mathrm{d} t=2 \pi a^{3 / 2} / \sqrt{G M}$, in the form

$t=\frac{2 \pi}{K \sqrt{G M}} \frac{\Sigma_{*}}{\Sigma_{\odot}} \int_{x_{0}}^{x} \frac{a(\bar{x})^{3 / 2}\left[a(\bar{x}) y(\bar{x}) / r_{\mathrm{g}}\right]^{-s} \mathrm{~d} \bar{x}}{\sqrt{(3-y(\bar{x})-2 \bar{x})\left(1-\bar{x}^{2}\right)}}$.

Here, a factor missing in Eq. (19) of Šubr \& Karas (1999) is corrected (no other equations and graphs were affected by that omission). The orbital decay manifests itself in the gradual decrease of $a, e$ and $i$, for which surface density of the disc is the main factor. The time derivative of the semi-major axis is

$\dot{a}_{\mathrm{col}}=\mathcal{B} y^{-q_{4}}\left[\frac{\Sigma_{*}}{\Sigma_{\odot}}\right]^{-1} \sqrt{\frac{3-y-2 x}{y\left(1-x^{2}\right)}}(2-x-y)$,

where

$\mathcal{B}=-B c M_{8}^{q_{1}} \mu_{\mathrm{E}}^{q_{2}}\left[\frac{\alpha}{0.1}\right]^{q_{3}}\left[\frac{a}{r_{\mathrm{g}}}\right]^{-q_{4}}$

and $\mu_{\mathrm{E}} \equiv \dot{M} /\left(0.1 \dot{M}_{\mathrm{E}}\right)$ is the accretion rate in units of Eddington accretion rate (with a $10 \%$ efficiency factor introduced). The factor $B$ and power-law indices $q_{1 \ldots 4}$ are determined by details of the particular model adopted to quantify the disc properties. Table 1 gives the values relevant for different regions of the standard disc as well as for the gravitationally unstable outer region (Collin \& Huré 1999). Notice that the algebraic functional form of radial dependencies remains identical in all these cases (a powerlaw), and we can use it with convenience also later for different prescriptions of the satellite-disc encounters.

\subsection{An orbiter embedded in the disc}

As drag is exerted on the satellite body, its orbit becomes circular and declined in the disc plane. Within this framework, orbital eccentricity and inclination are expected to
Table 1. Parameters in Eqs. (5), (7) and (9) describing the orbital decay in the case of different regimes and for different disc models.

\begin{tabular}{cl|lcccc}
\hline Regime & Disc & $B$ & $q_{1}$ & $q_{2}$ & $q_{3}$ & $q_{4}$ \\
\hline col & (i) & $2.3 \times 10^{-9}$ & 0 & -1 & -1 & -1 \\
col & (ii) & $2.9 \times 10^{-5}$ & $1 / 5$ & $3 / 5$ & $-4 / 5$ & $11 / 10$ \\
col & (iii) & $1.1 \times 10^{-4}$ & $1 / 5$ & $7 / 10$ & $-4 / 5$ & $5 / 4$ \\
col & (iv) & 4.05 & $-9 / 7$ & $1 / 7$ & 0 & $37 / 14$ \\
col & (v) & $1.1 \times 10^{-2}$ & -1 & $1 / 9$ & 0 & $13 / 6$ \\
gap & (i) & $1.3 \times 10^{-3}$ & 0 & 2 & 1 & $5 / 2$ \\
gap & (ii) & $7.4 \times 10^{-8}$ & $-1 / 5$ & $2 / 5$ & $4 / 5$ & $2 / 5$ \\
gap & (iii) & $2.8 \times 10^{-8}$ & $-1 / 5$ & $3 / 10$ & $4 / 5$ & $1 / 4$ \\
gap & (iv) & $6.1 \times 10^{-13}$ & $9 / 7$ & $6 / 7$ & 0 & $-8 / 7$ \\
gap & (v) & $2.2 \times 10^{-10}$ & 1 & $8 / 9$ & 0 & $-2 / 3$ \\
dw & (i) & $1.8 \times 10^{-18}$ & 0 & -3 & -1 & -5 \\
dw & (ii) & $1.3 \times 10^{-10}$ & $2 / 5$ & $1 / 5$ & $-3 / 5$ & $-4 / 5$ \\
dw & (iii) & $4.8 \times 10^{-9}$ & $2 / 5$ & $2 / 5$ & $-3 / 5$ & $-1 / 2$ \\
dw & (iv) & $8.2 \times 10^{-7}$ & $-5 / 7$ & $-1 / 7$ & 0 & $5 / 14$ \\
dw & (v) & $2.9 \times 10^{-4}$ & -1 & $-1 / 9$ & 0 & $5 / 6$ \\
\hline
\end{tabular}

Notation used in table.

Disc models: (i) ... Standard disc with $p=p_{\text {rad }}, s=3 / 2$; (ii) $\ldots$ Standard disc $p=p_{\text {gas }}, s=-3 / 5$, electron scattering opacity; (iii) ... The same as (ii) but with $s=-3 / 4$ and free-free opacity; (iv) ... Marginally unstable self-gravitating disc (solar metallicity, optically thick), $s=-15 / 7$; (v) ... The same as (iv) but for zero metallicity, optically thin medium, $s=-5 / 3$; cf. Sect. 3 for further details.

Regimes of orbital decay: Orbital decay dominated by stardisc collisions (col), by gap formation in the disc (gap), and by density waves $(\mathrm{dw})$, respectively.

reach zero values in the disc, so that quasi-circular trajectories are relevant near the center.

The orbit evolution is thus reduced to the situation which was addressed by several people (e.g., King \& Done 1993; Takeuchi et al. 1996; Ward 1997; Ostriker 1999) in connection with formation and subsequent migration of bodies inside the disc. Two basic modes can be distinguished according to the disc properties and the orbiter mass. First, a gap is cleared in the disc if the satellite's Roche radius exceeds the disc thickness, $r_{\mathrm{L}} \approx\left(M_{*} / M\right)^{1 / 3} r \gtrsim h$, and simultaneously $M_{*} \gtrsim M_{\text {gap }} \approx \sqrt{40 \alpha} \epsilon^{5 / 2} M$ (Lin \& Papaloizou 1986). Motion of the satellite is then coupled with the disc inflow, so that

$\dot{a}_{\text {gap }}=\mathcal{B}$.

On the other hand, if the satellite is unable to create the gap, the gas drag is imposed on it through quasispherical accretion. The resulting radial drift is weaker by a factor $\epsilon \ll 1$ than the drift caused by density-wave excitation (Ward 1986; Artymowicz 1994). Hence, such a 


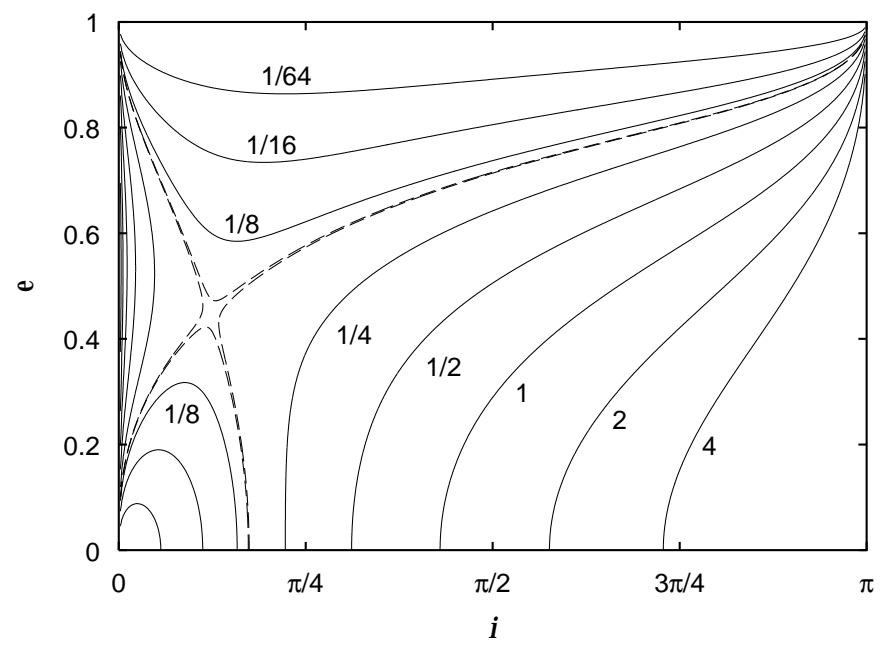

Fig. 1. Contours of $f$ are plotted as a function of inclination and eccentricity for the case (ii). Function $f(i, e)$ characterizes the relative importance of energy losses in Eq. (16). Values of $f$ are indicated with contour lines. A saddle-type point develops between two neighbouring contours, $f=0.150$ and $f=0.152$, which are plotted with dashed lines. Its exact location depends on the disc model but the overall picture remains very similar in other cases, too.

satellite migrates inward mainly due to the latter effect on the time-scale

$t_{\mathrm{dw}}=\left(C M_{*} \Omega\right)^{-1} \epsilon^{2}\left(\frac{M^{2}}{\pi r^{2} \Sigma_{\mathrm{d}}}\right)$,

where $C$ is a dimensionless constant of the order of unity. Using Eq. (8) and $\mathrm{d} a / \mathrm{d} t \approx r / t_{\mathrm{dw}}$ we obtain

$\dot{a}_{\mathrm{dw}}=\frac{M_{*}}{M_{\odot}} \mathcal{B}$.

Substantial differences in the satellite migration are thus introduced in the model already within this very simplified picture where the process of satellite sinking is driven by the gas medium. For the region of the gas pressure dominated standard disc and for a self-gravitating zerometalicity model $(\mathrm{v})$, the chance of opening the gap increases with decreasing radius. The situation is opposite in the case (i) (radiation pressure dominated) and for the solar-metallicity disc (iv).

\subsection{The orbital decay due to gravitational radiation}

The orbiting companion emits continuous gravitational radiation whose waveforms are of particular relevance for gravitational wave searches from compact binaries in the Milky Way. Possible approaches to their observational exploration have been discussed by several people (e.g., Nakamura et al. 1987; Dhurandhar \& Vecchio 2001; Hughes 2001). The average rate of energy loss which the orbiter experiences via gravitational radiation over one revolution can be written in terms of orbital parameters (Peters \& Mathews 1963),

$$
\dot{E}_{\mathrm{gw}}=\frac{32}{5} \frac{G^{4}}{c^{5}} \frac{M^{3} M_{*}^{2}}{a^{5}\left(1-e^{2}\right)^{7 / 2}}\left(1+\frac{73}{24} e^{2}+\frac{37}{96} e^{4}\right) .
$$

Corresponding to Eq. (10) are the change of semi-major axis

$$
\begin{aligned}
\dot{a}_{\mathrm{gw}}= & -1.28 \times 10^{-7} c M_{8}^{-1} \frac{M_{*}}{M_{\odot}}\left[\frac{a}{r_{\mathrm{g}}}\right]^{-3} \\
& \times \frac{1+\frac{73}{24} e^{2}+\frac{37}{96} e^{4}}{\left(1-e^{2}\right)^{7 / 2}} .
\end{aligned}
$$

and the loss of angular momentum

$$
\dot{L}_{\mathrm{gw}}=\frac{32}{5} \frac{G^{7 / 2}}{c^{5}} \frac{M^{5 / 2} M_{*}^{2}}{a^{7 / 2}\left(1-e^{2}\right)^{2}}\left(1+\frac{7}{8} e^{2}\right) .
$$

The above formulae (10)-(12) assume that the satellite star follows an eccentric orbit in Schwarzschild geometry of the central massive body. Gravitational radiation losses compete with those caused by star-disc encounters. We are thus interested in the relative importance of these mechanisms, which is characterized by the ratio $\mathcal{R}_{\mathrm{col} / \mathrm{gw}}=[\mathrm{d} a / \mathrm{d} t]_{\mathrm{col}} /[\mathrm{d} a / \mathrm{d} t]_{\mathrm{gw}}$ :

$\mathcal{R}_{\mathrm{col} / \mathrm{gw}}=\frac{5 K}{32 \pi} \frac{M}{M_{*}}\left[\frac{\Sigma_{*}}{\Sigma_{\odot}}\right]^{-1}\left[\frac{a}{r_{\mathrm{g}}}\right]^{s+5 / 2} f(x, e)$,

where, for whichever of the models described by the powerlaw density profile,

$f(x, e)=\frac{\left(1+e^{2}-x\right)\left(1-e^{2}\right)^{s+5 / 2}}{1+\frac{73}{24} e^{2}+\frac{37}{96} e^{4}} \sqrt{\frac{2+e^{2}-2 x}{1-x^{2}}}$.

For the standard thin disc model (i) we obtain

$$
\begin{aligned}
\mathcal{R}_{\mathrm{col} / \mathrm{gw}}^{(\mathrm{i})} \approx & 1.8 \times 10^{-2} M_{8} \mu_{\mathrm{E}}^{-1}\left[\frac{\alpha}{0.1}\right]^{-1} \\
& \times\left[\frac{\Sigma_{*}}{\Sigma_{\odot}}\right]^{-1}\left[\frac{M_{*}}{M_{\odot}}\right]^{-1}\left[\frac{a}{r_{\mathrm{g}}}\right]^{4} f(x, e),
\end{aligned}
$$

while for a gas pressure dominated disc (ii)

$$
\begin{aligned}
& \mathcal{R}_{\mathrm{col} / \mathrm{gw}}^{(\mathrm{ii})} \approx 1.5 \times 10^{2} M_{8}^{6 / 5} \mu_{\mathrm{E}}^{3 / 5}\left[\frac{\alpha}{0.1}\right]^{-4 / 5} \\
& \times\left[\frac{\Sigma_{*}}{\Sigma_{\odot}}\right]^{-1}\left[\frac{M_{*}}{M_{\odot}}\right]^{-1}\left[\frac{a}{r_{\mathrm{g}}}\right]^{29 / 10} f(x, e)
\end{aligned}
$$

For the model (iii) we find

$$
\begin{aligned}
\mathcal{R}_{\mathrm{col} / \mathrm{gw}}^{(\mathrm{iii})} \approx & 8.6 \times 10^{2} M_{8}^{6 / 5} \mu_{\mathrm{E}}^{7 / 10}\left[\frac{\alpha}{0.1}\right]^{-4 / 5} \\
& \times\left[\frac{\Sigma_{*}}{\Sigma_{\odot}}\right]^{-1}\left[\frac{M_{*}}{M_{\odot}}\right]^{-1}\left[\frac{a}{r_{\mathrm{g}}}\right]^{7 / 4} f(x, e) .
\end{aligned}
$$

We show the functional form (14) in Fig. 1 where the factor $f(x, e)$ determining the dependence of $\mathcal{R}_{\mathrm{col} / \mathrm{gw}}$ on inclination and eccentricity is plotted for the case (16). In other words, $f(x, e)$ represents that part of the drag ratio that 
is independent of the disc medium and the satellite physical properties; only the two mentioned orbital parameters play a role here. Typically, for a solar-type star it is only on eccentric orbits that $f$ becomes small enough to bring $\mathcal{R}_{\text {col/gw }}$ below unity. The required eccentricity is rather high, and such a satellite would be trapped or disrupted directly by the central hole. Otherwise, $\mathcal{R}_{\text {col/gw }} \gg 1$ for $a \gtrsim r_{\mathrm{g}}$ and for standard values of the disc parameters $\left(\alpha \lesssim 1, \mu_{\mathrm{E}} \approx 1\right)$. This means that direct hydrodynamical interaction with the disc plays a dominant role in the orbital evolution of satellites crossing the disc, unless the medium is extremely rarefied (e.g., an ADAF; Narayan $2000)$. Notice that the point $f(1,0)=0$ (i.e. a fully circularized orbit inclined into the disc plane) is the exception, where the adopted approximation of instantaneous collisions breaks down.

Analogous to $\mathcal{R}_{\mathrm{col} / \mathrm{gw}}$, one could explore the relative ratio of the hydrodynamical versus gravitational radiation losses in other regimes of the satellite-disc interaction. In this way, the relevant formulae (those which apply in the course of orbiter evolution) are

$$
\begin{aligned}
\mathcal{R}_{\mathrm{dw} / \mathrm{gw}} & =2.3 \times 10^{-12} M_{8} \mu_{\mathrm{E}}^{-3}\left[\frac{\alpha}{0.1}\right]^{-1}\left[\frac{a}{r_{\mathrm{g}}}\right]^{8}, \\
\mathcal{R}_{\mathrm{gap} / \mathrm{gw}} & =5.2 \times 10^{4} M_{8} \mu_{\mathrm{E}}^{2}\left[\frac{\alpha}{0.1}\right]^{-1}\left[\frac{M_{*}}{M_{\odot}}\right]^{-1}\left[\frac{a}{r_{\mathrm{g}}}\right]^{1 / 2},
\end{aligned}
$$

for the inner, radiation pressure dominated disc (i), and

$$
\begin{aligned}
& \mathcal{R}_{\mathrm{dw} / \mathrm{gw}}=1.0 \times 10^{-4} M_{8}^{7 / 5} \mu_{\mathrm{E}}^{1 / 5}\left[\frac{\alpha}{0.1}\right]^{-2 / 5}\left[\frac{a}{r_{\mathrm{g}}}\right]^{19 / 5}, \\
& \mathcal{R}_{\mathrm{gap} / \mathrm{gw}}=6.3 M_{8}^{4 / 5} \mu_{\mathrm{E}}^{2 / 5}\left[\frac{\alpha}{0.1}\right]^{4 / 5}\left[\frac{M_{*}}{M_{\odot}}\right]^{-1}\left[\frac{a}{r_{\mathrm{g}}}\right]^{13 / 5}
\end{aligned}
$$

for the middle region (ii). Here, the eccentricity-dependent factor was omitted upon the finding that orbits are almost circular when inclined in the plane of the disc. It is evident from Eqs. (13)-(21) that gravitational radiation can have a visible impact only at small $a$, especially when Eq. (18) applies (cp. also Fig. 5 and related discussion below).

\subsection{Evolutionary tracks of the satellite}

Now we explore the evolutionary tracks of the satellite in the parameter space of osculating elements. We start by considering two effects: gravitational-wave losses in the approximation of Eqs. (10)-(12), and hydrodynamical drag acting on the satellite according to Eq. (1) twice per revolution. Dissipation operates with an efficiency depending on the type of satellite, and it provides a mechanism for the separation of different types of bodies in the phase space of a cluster.

Typical results of orbit integrations are presented in Fig. 2. Notice the big difference in time-scales relevant for non-compact stars (left panel) when compared with compact ones (right panel). In the former case, hydrodynamical drag is more pronounced. It gradually changes the orbital plane, while gravitational radiation can be safely

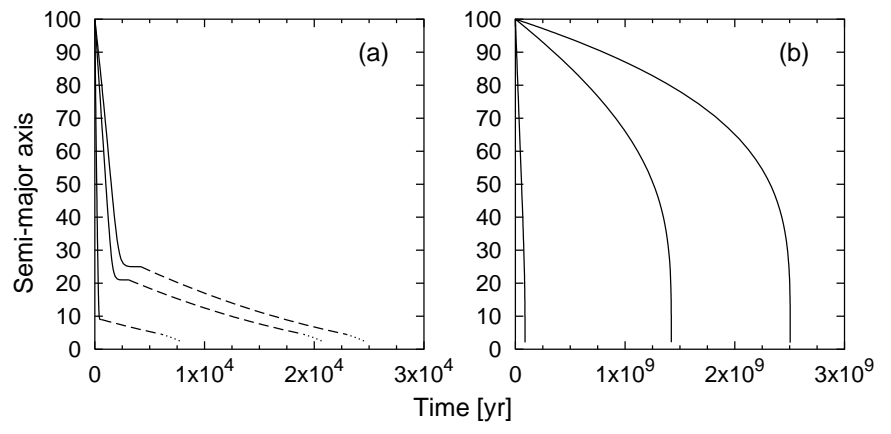

Fig. 2. Evolutionary tracks of the satellite are plotted in the plane semimajor axis $a$ (units of $r_{\mathrm{g}}$ ) versus time (years). Two very different cases are shown. a) Left: solar-type satellite $\left(\Sigma_{*}=\Sigma_{\odot}\right)$. The three curves correspond to different initial eccentricities (from top to bottom), $e_{0}=0,0.4$, and 0.8. Each curve is divided into three segments. Solid line indicates the period of motion outside the disc (when collisions occur with large orbital inclination); dashed line corresponds to densitywave driven motion in the disc; dotted line is the late stage with gap formation. b) Right: a compact satellite $\left(\Sigma_{*}=10^{7} \Sigma_{\odot}\right)$. Only the first stage is resolved here. In all cases, $a_{0}=100$, $x_{0}=0$. Gravitational radiation contributes to orbital changes independent of inclination but its impact is clearly important near the centre, where $a$ decreases rapidly.

neglected. The satellite sinks in the disc where the impulsive approximation (5) loses its validity and it is substituted by motion in the disc plane. Time-scales are generally longer in the latter, compact satellite case, although gravitational wave emission speeds up the evolution at very late stages $(a \lesssim 50)$. For definiteness, we adopt the disc model (iii) and the condition of $\tan i_{\mathrm{tr}}=h / r$ when the transition in the disc occurs. We checked by modifying the value of $\tan i_{\mathrm{tr}}$ by a factor of 10 that the qualitative picture of orbital evolution does not depend on its exact choice and also that numerical results remain similar.

\subsection{Limitations of the adopted approximations}

For simplification, we ignored the effects of gradual change of the mass of both the satellite and the central body. Furthermore, we assumed that the interaction has no effect on the structure of the satellites. This is a plausible assumption for stars with column densities much larger than that of the disc, while it is inadequate for giants which must quickly lose their atmospheres (Armitage et al. 1996). Also, we did not consider various effects acting on the disc structure (e.g. torques imposed on it by the dense cluster of stars; Ostriker 1983). Although all these effects will be important for a complete unified treatment of accreting black holes in active galactic nuclei, they can be neglected without losing the main physical effects influencing the satellite motion in the present simplified scheme.

We note that the significance of direct orbiter's collisions with the disc material is controlled by a characteristic time-scale $\tau \propto \Sigma_{*} / \Sigma_{\mathrm{d}}(r)$, which we expressed in terms of the orbital decay $\dot{a}$. Let us recall that dimensionless parameter characterizing compactness of the 
orbiter can be introduced in different ways. While $\Sigma_{*} / \Sigma_{\mathrm{d}}$ stands directly in the description of star-disc collisions, the usual factor $\varepsilon=G M_{*} R_{*}^{-1} c^{-2}$ determines the importance of general relativity effects near the surface of a compact body: $\varepsilon \approx 10^{-6}\left(\Sigma_{*} / \Sigma_{\odot}\right)\left(R_{*} / R_{\odot}\right)$. We treat motion in the Newtonian regime and we only take the possibility of the satellite capture into account by removing the orbiter from a sample if its trajectory plunges too close to the central mass, below a marginally stable orbit. Another dimensionless quantity has also been designated as the compactness parameter when describing accretion onto compact objects, $\tilde{\varepsilon}=L \sigma_{\mathrm{T}} r^{-1} m_{\mathrm{e}}^{-1} c^{-3}$. It considers the effect of radiation luminosity $L$ acting through a cross-section $\sigma_{\mathrm{T}}$ in the medium, however, we can safely ignore radiation pressure on macroscopic satellites hereafter.

The gradual and monotonic decrease of eccentricity is overlaid with short-term oscillations if the disc mass is non-negligible (Vokrouhlický \& Karas 1998). Also the satellites' inclination converges to a somewhat different distribution (instead of a strictly flattened disc-type system) when two-body gravitational relaxation is taken into account (Šubr 2001; Vilkoviskiy 2001 - preprint). On the other hand, complementary to the scenario of the satellites grinding into the disc is the picture of enhanced star formation in the disc plane, in which case the stars are born with zero inclination (Collin \& Zahn 1999). But these effects, as well as evaporation processes operating in the cluster, as suggested by various Fokker-Planck simulations (e.g., Kim et al. 1999), remain beyond the scope of the present paper.

We could see that different mechanisms (of which we considered particular examples) affect the orbital motion rather selectively, depending on the orbiter's size and mass. One thus expects separation of different objects in the cluster phase space. In order to verify this expectation we examine in the following paragraph a simple scheme which captures gradual changes in the structure of the cluster. Such a discussion is required: indeed, in the absence of sufficient resolution which would enable tracking of individual stellar paths in nuclei of other galaxies, one needs to inspect the overall influence on the members of the cluster, namely, the change of the radial distribution of the satellites in terms of their number fraction and average inclination.

Let us note that the accretion flow is supposed to remain undisturbed by the presence of the embedded cluster. This assumption gives an upper limit on the total number of stars inside the radius $r_{\mathrm{d}}\left(\approx 10^{4} r_{\mathrm{g}}\right)$, and on the fraction of those dragged into the disc plane in this region. A simple smooth disc can be destroyed, especially in the process of gap formation (the case of sufficiently large $M_{*}$ and small $h$ ); the models (i) and (v) are particularly susceptible to the occurence of multiple gaps. Very roughly, if $\approx 2 \%$ of the total number of $N=10^{4}$ satellites get aligned with the disc at late times (a result of our computations for the model (iii)), then their Roche lobes might cover an area of the order $\approx 0.01 N r_{\mathrm{d}} r_{\mathrm{L}}$. This is just comparable with the total disc surface for $M_{*} \approx 1 M_{\odot}$, however, recall that there are more conditions for the gap formation depending on the disc model. Also, effects of two-body relaxation and of satellite scattering in the gravitational potential of the disc are expected to decrease the fraction of aligned bodies when these effects are taken into account.

\section{The cluster evolution}

We follow the cluster evolution starting from a clean stationary state (no dissipative perturbation), which corresponds to a spheroidal stellar system gravitationally coupled with the central black hole (Bahcall \& Wolf 1976). Hence, number density $n_{0}(a)$ of the satellites is assumed proportional to $a^{1 / 4}$, while the initial distribution in eccentricities and inclinations conforms with the condition of a randomly generated sample. Common parameters of the computations are the mass $M=10^{8} M_{\odot}$ of the central body, and the orbiters' column density $\Sigma_{*}=\Sigma_{\odot}$. Now, the perturbation effects are switched on, depending on the prescribed disc type and accretion rate (typically $\mu_{\mathrm{E}}=1$, but we also examined other situations and less conventional values of the above parameters; cf. Šubr 2001).

As a consequence of the dissipative action, the system departs from the initial configuration. We concentrate our main attention on the range of radii $r \lesssim 10^{3} r_{\mathrm{g}}$, well below the outer edge of the disc $r_{\mathrm{d}} \approx 10^{4} r_{\mathrm{g}}$ (where $\Sigma_{\mathrm{d}}$ becomes negligible). We find a new quasi-stationary, modified cluster distribution developing in that region. On the outer boundary, fresh satellites could be inserted in the system from an external reservoir in order to maintain the steady flow towards the center, but in this work we alternatively consider a closed system with a large but finite number of its members, $N_{0}$, which are not replenished. The actual value of $N_{0}$ is not very important in the present work because we neglect gravitational interaction among the satellites themselves ( $N_{0}$ will play a role when two-body collisions are taken into account).

\subsection{A role of the disc model}

Several different models of the disc were adopted. We specify them in terms of their corresponding density profiles, $\Sigma_{\mathrm{d}}(r)$, and vertical thicknesses, $h(r)$ :

(i) Radiation pressure dominated, geometrically thin disc (Shakura-Syunaev $\alpha$-viscosity model; Frank et al. 1992);

(ii) Gas pressure dominated standard disc with opacity due to electron scattering;

(iii) The same as in (ii) but with opacity dominated by free-free scattering;

(iv) Gravitationally unstable discs in the region of a self-gravitating disc beyond the Toomre radius; see Sect. (2.3) of Collin \& Huré (1999) for analysis of the marginally unstable $(\zeta=5)$ self-gravitating disc with solar metallicity and large optical thickness; 


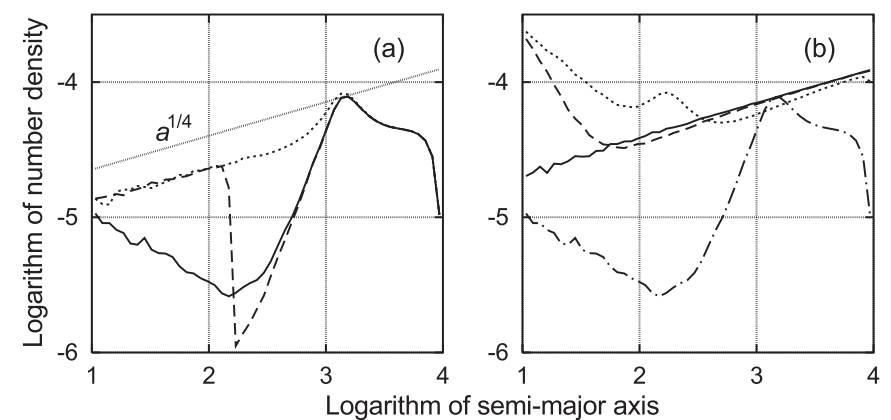

Fig. 3. Number densities $n_{t}(a)$ of the cluster. Left panel: quasistationary states for $M_{*}=1 M_{\odot}$ (solid line), $3 M_{\odot}$ (dashed), and $10 M_{\odot}$ (dotted). Right panel: snapshots of $n_{t}(a)$ are shown for $M_{*}=1 M_{\odot}$ at $t=10^{6}$ (solid), $10^{8}$ (dashed), $10^{10}$ (dotted), and $10^{12}$ (dash-dotted). In all cases, $\Sigma_{*}=\Sigma_{\odot}$ (solartype compactness of the satellite). On the vertical axis, units are arbitrary and scaled with respect to the initial profile $n_{0}(a) \propto a^{1 / 4}$. Units of $G M / c^{2}$ are used for $a$ on the horizontal axis.

(v) The same as in (iv) but for optically thin medium with zero metallicity.

The choice of different models is dictated by their applicability for the description of accretion regimes relevant under different conditions, namely, the cases (iv)(v) are appropriate for black-hole discs at large $\left(\gtrsim 10^{3} r_{\mathrm{g}}\right)$ radii. One could consider other models relevant in various situations but the adopted examples cover qualitatively different evolutions which are frequently encountered. In particular, the above models comprise a range of $\Sigma_{\mathrm{d}} / \Sigma_{*}$ dependencies (dimensionless factor determining the evolutionary time-scales) and different $(h / r)\left(\Sigma_{\mathrm{d}} / \Sigma_{*}\right)=\epsilon / \tau$ whose radial dependence determines area in the disc where the gap is formed.

First, we carried out simulations while sticking to one of these models in the whole range of radii from the centre up to the outer edge, even if it extends somewhat out of the zone of validity of the particular model. This helps us to avoid additional complexities which could stem from a complicated prescription for the disc properties. For example, one expects a different rate of radial drift across boundaries where the disc properties are switched, but we refrain from this complication for the moment. This effect will be observed later on: to achieve a more realistic description, a further step will be carried out by joining different regions of the disc in a unified model. In that case, the radiation pressure supported standard disc might be limited to the innermost part of the system while gravitationally unstable part takes over at distances of the order of a few hundreds $r_{\mathrm{g}}$.

Parameters defining radial migration in the models (i)-(v) were given in Table 1 . Here we only remark that the factor $B$ determines the pace in absolute time units with which the evolution proceeds, while power-law indices $q_{i}$ influence the form of the satellites number density distribution $n(a)$.

\subsection{Modified cluster distributions}

The main results are shown in Fig. 3, in which the disc model (iii) is adopted. This will serve as a representative test case (more examples are deferred to Appendix). It is particularly interesting to observe transition regions where the mode of the mutual satellite-disc interaction is changed. At the transition, the efficiency of radial transfer of the satellites is changed, and this effect produces sudden changes (either dips or concentrations) in $n_{t}(a)$ profiles. If the average inclination of the satellites is small (the case of a flattened configuration), a ring is created in the place where the orbital decay is stalled.

The computations were launched from the initial distribution $n_{0}(a) \propto a^{1 / 4}$. In the left panel, Fig. 3a, new $n_{t}(a)$ profiles are shown at a sufficiently late time, $t=10^{12}$, when a quasi-stationary state has been established $^{1}$. Three cases are plotted with increasing satellite masses: $M_{*}=1 M_{\odot}, 3 M_{\odot}$, and $10 M_{\odot}$. After being inclined to the disc, low mass satellites proceed via density waves (e.g., $1 M_{*}$ case with $n_{t}(a) \propto a^{-1 / 2}$ at small $a$ ). On the other hand, very massive satellites develop a gap already very far from the centre and they proceed in this mode the whole way down $\left(10 M_{*}\right.$ case with $n_{t}(a) \propto a^{1 / 4}$, which is incidentally parallel with the initial $n_{0}$ distribution $)^{2}$. Intermediate satellites show an evident dip in $n_{t}(a)$ : its formation is connected with the gap in the disc which arises at sufficiently small $a$. Exact location of the dip depends on the disc model too; for $3 M_{\odot}$ satellites it occurs at $a \lesssim 150$.

The right panel, Fig. 3b, is complementary to the left one, showing temporal evolution of $n_{t}(a)$ for one solar mass satellites. One notices two areas with different $n_{t}(a)$ slopes in the graph: at small $a$ the overall distribution is eventually dominated by satellites residing in the disc (corresponding to negative slope), while a whole mixture of satellite inclinations persists farther out (a positive slope). Transition between the two regions occurs at $a \approx 10^{2}$ (its location moves gradually towards larger $a$ as time proceeds; see the figure). A terminal dip is seen at large $a$ (on the right of the graphs); it is caused by depletion of the sample in the simulation.

A realistic situation must involve further changes of the $n_{t}(a)$ slope caused by varying the accretion mode (see Appendix and Fig. A.1 for details). In this respect, the form of quasi-stationary distributions is of particular interest (the persisting quasi-stationary profile of $n_{t}(a)$ is given more detailed discussion in the next section, Sect. 3.3). We remark that the long-term evolution of the

\footnotetext{
1 Time is given in units $G M=c=1$, total number of satellites is $N=\int n_{t}(a) \mathrm{d} a$. Conversion of time $t$ to physical units is achieved by $t_{\mathrm{phys}}[\mathrm{yr}]=1.6 \times 10^{-5} M_{8} t$, while for distances $a_{\text {phys }}[\mathrm{cm}]=1.5 \times 10^{13} M_{8} a$.

${ }_{2}$ The values of $n_{t}(a)$ slopes are further explained in Sect. 3.3. We recall that these slopes refer to the motion in the disc. The overall $n_{t}(a)$ profiles can be thus different in the region where the whole sample is dominated by those satellites moving along inclined trajectories outside the disc.
} 


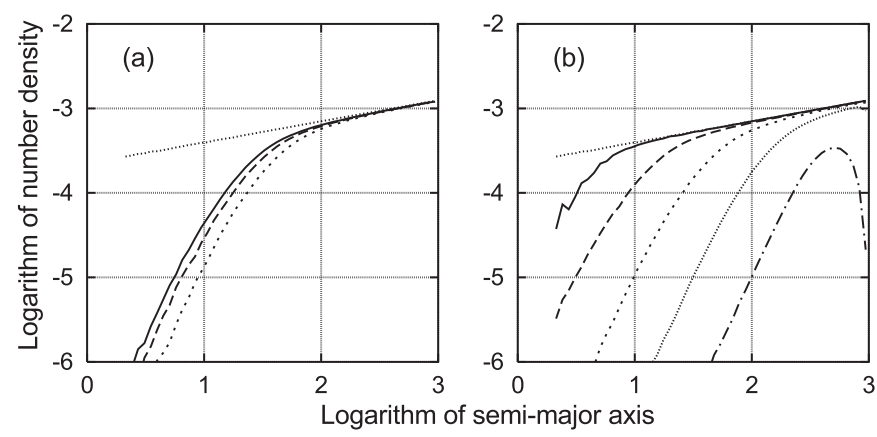

Fig. 4. Left panel: the shape of the distribution $n_{t}(a)$ of a cluster containing compact orbiters of the masses $M_{*}=1.5 M_{\odot}$ (solid line), $3 M_{\odot}$ (dashed) and $10 M_{\odot}$ (dotted) at $t=10^{12}$. Right panel: subsequent phases of evolution for a cluster of neutron stars with $M_{*}=1.5 M_{\odot}$. The power-law is represented by a line in the log-log plane of $n_{t}$ (normalized by $N_{0}$, the initial number of cluster members) vs. $a$ (units of $r_{\mathrm{g}}$ ). Again, a thin dotted line stands for the initial power-law distribution, $n_{0}$. Other curves correspond, from top to bottom, to $t=10^{9}, 10^{11}$, $10^{13}, 10^{15}$, and $10^{17}$.

orbits is influenced by collisions with the disc especially if the satellite's cross-sectional area is large, e.g. a giant star or a solar-size body, but quite the opposite conclusion can be drawn for compact objects such as neutron stars and stellar-mass black holes for which the cross-section is much smaller, diminishing their collisional interaction with the disc. The whole cluster is modified by this kind of dissipative process in a selective manner, depending on $M_{*}$.

In Fig. 4a we present the resulting form of the distribution $n_{t=10^{12}}(a)$ for different masses of compact bodies. On the other hand, panel $4 \mathrm{~b}$ concerns a cluster consisting of one solar-mass satellites, $M_{*}=1 M_{\odot}$, shown at different times (several consecutive moments are examined with a logarithmic spacing in $t$ ). Now, there is no need to consider separately that part of the distribution of trajectories inclined into the disc; indeed, no compact bodies reach the disc plane, even at late phases of the evolution. This implies that the orbits evolve predominantly by gravitational radiation and the resulting graphs come out almost identical for all the disc models under consideration. We are thus led to restrict ourselves to non-compact satellites which suffer relatively intense drag from the disc.

\subsection{A quasi-stationary state}

Time-scales of the perturbed cluster evolution depend on the disc model and, as shown above, a number of other factors characterizing the cluster members - their typical mass, compactness and efficiency of the dissipative interaction with the disc medium. With solar-type (and less compact) satellites, the disc plays a dominant role in determining the form of $n_{t}(a)$. The starting canonical distribution $n_{0}(a) \propto a^{1 / 4}$ (corresponding to $\rho \propto r^{-7 / 4}$ in the notation of Bahcall \& Wolf 1976) changes to $n_{t}(a)$, where it stands almost frozen in a quasi-stationary state for a prolonged interval of time. This is true also in the case of

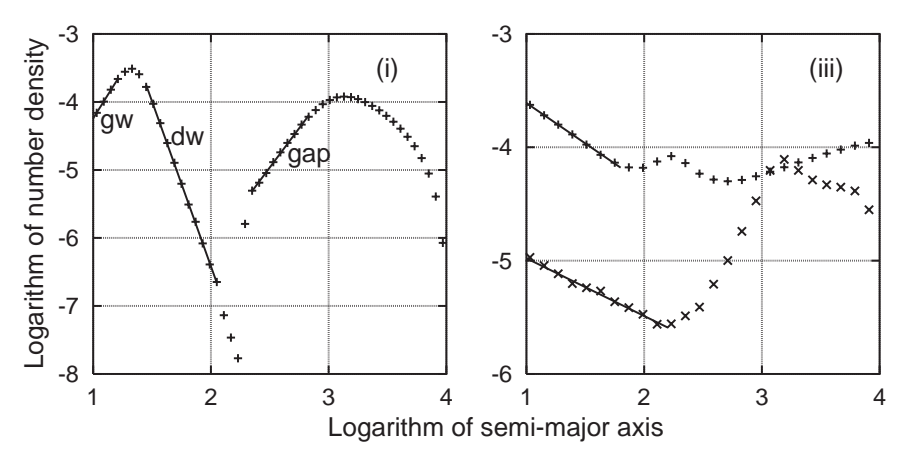

Fig. 5. Left panel: a quasi-stationary $n_{t}(a)$ profile is plotted (by crosses) for the model (i), $t=10^{13}$. A power-law approximation, $n_{t}(a) \propto a^{q_{\mathrm{f}}}$, is constructed in appropriate ranges of radius (solid lines). Suppression of the profile occurs at small $a$ in consequence of quite efficient gravitational radiation losses near the center. Right panel: the slope evolves from the initial value, $q=1 / 4$, until the quasi-stationary state of $q \rightarrow q_{\mathrm{f}}$ is reached. Two snapshots are shown for the model (iii): $t=10^{10}$ (upper profile), and $t=10^{12}$ (lower profile). See the text for corresponding values of $q_{\mathrm{f}}$.

initial profiles reasonably different from the canonical one. In particular, we verified that $n_{0}(a)=$ const. converges to identical slopes as those shown in Fig. A.1 at $t \approx 10^{12}$ (cf. the bottom row of plots in the Appendix).

Clear-cut results emerge provided the distribution is influenced by a single mode of star-disc interaction. The evolution time-scales are shorter in the region where substantial fraction of the satellites are already inclined into the disc plane, so that $n_{t}(a)$ forgets about the exact initial distribution much earlier, at about $t \gtrsim 10^{8}-10^{9}$. In that case the final slope $q_{\mathrm{f}}$ ranges from the initial value up to the limiting value $q_{4}$ of the corresponding model and the mode of migration (the values are listed in Table 1). The actual final value of $q_{\mathrm{f}}$ reaches $q_{4}$ if two conditions are satisfied in the region where the power-law distribution is established: first, the flow of the satellites in this region continues in the disc plane (all bodies are inclined to the disc); second, the satellites inflow is faster than the changes of their number density at the outer boundary. These conditions are satisfied in the late stages until the sample is exhausted. In other words, the modified cluster distribution reflects the adopted disc model while it is rather insensitive to the initial form of $n_{0}(a)$.

This behaviour is illustrated in Fig. 5 in terms of a power-law approximation to the computed distributions. Given the disc model, different power-law relations may be established in separate regions depending on the mode of star-disc interaction applicable at that distance. In the case (i) and for solar-type satellites, we find that the slope $q_{\mathrm{f}}=-4.9$ arises in the region $30 \lesssim a \lesssim 120$ (the evolution is driven by density waves, accumulating the satellites in an evident peak), while $q_{\mathrm{f}}=2.3$ suits for $220 \lesssim a \lesssim 680$ (i.e. beyond the transition point where the star-disc interaction is determined by the gap; here, as an example, $q_{\mathrm{f}}=2.3$ is to be compared with the corresponding $q_{4}=5 / 2$ in the sixth row in Table 1). Furthermore, the slope is 
influenced by gravitational radiation which dominates the evolution on very small radii: $q_{\mathrm{f}}=2.8$ for $r \leq 20$. The three regions are distinguished in the graph: gw (dominated by gravitational waves), dw (density waves), gap (gap formation). The large span of values acquired by $q_{\mathrm{f}}$ reflects the presence of ripples in $n_{t}(a)$ which develop in certain areas of the disc.

The right panel of Fig. 5 shows how the $n_{t}(a)$ profile is gradually adjusted as time proceeds. Here we plot the case (iii) with $M_{*} \lesssim 1 M_{\odot}$ for illustration, and we find $q=-0.71\left(t=10^{10}, 10 \lesssim r \lesssim 60\right)$, and $q=-0.51$ $\left(t=10^{12}, 10 \lesssim r \lesssim 160\right)$, respectively. On the vertical axis, one can read the fraction of satellites with semi-major axes falling in interval $\langle a, a+\mathrm{d} a\rangle$. One observes different slopes for more massive satellites, $M_{*} \gtrsim 3 M_{\odot}$, because of gap formation (the slope reaches $q \doteq 0.25$ ); cp. with Fig. 3 to see the effect of $M_{*}$. On the other hand, the situation gets simpler with the other models where such a transition does not occur, and a single power-law relation prevails at late stages for all $M_{*} \lesssim 10 M_{\odot}$. The emerging slopes are: (ii) $q_{\mathrm{f}}=-0.79$, and (iv) 0.30 , for which we found that the quasi-stationary state is established at $r \lesssim 200$, $t=10^{12}$. The case $(\mathrm{v})$ develops more slowly and reaches the expected value $q_{\mathrm{f}}=-0.67$ somewhat later $^{3}$, at time $t=10^{13}$.

\section{Conclusions}

Energy and angular-momentum losses of a satellite colliding with an accretion disc were examined. We explored how the relative importance of several secular effects depends on the parameters of the model, namely, the osculating elements of the satellite trajectories, surface density of the disc, and the mass and compactness of the orbiter bodies. We verified within the thin-disc analysis that hydrodynamical drag is, typically, more important for the long-term orbital evolution than gravitational radiation losses. Decay of the orbit due to interaction with the gaseous environment brings the orbiter gradually towards the center. This drag acts at different regimes depending on parameters of the orbiters and of the disc. The magnitude of the drag determines the rate of orbital decay and it influences the resulting structure of the cluster, especially the characteristic slope of $n_{t}(a)$ density distribution.

Gravitational radiation losses dominate over the hydrodynamical dissipation if the disc has low density and/or the orbiter is of very high compactness (a neutron star or a black hole). The two influences are thus complementary and they can operate simultaneously at different regions of a given source, assuming the thin-disc scheme is valid farther away from the center, whereas advectiondominated flow takes over at distances of the order of $\approx 10^{2} r_{\mathrm{g}}$. One could thus expect the final stages of the orbital decay to be governed by emission of gravitational

\footnotetext{
3 The expected final value of $q_{\mathrm{f}}$ for the case $(\mathrm{v})$ coincides with $q_{4}=-2 / 3$, as can be seen in the corresponding section (gap) of Table 1.
}

waves and by tidal interaction, while the initial transport from the outer cluster is ensured by other causes, possibly the hydrodynamical collisions together with dynamical friction. Our results are still only indicative in this part because several effects were ignored which must be taken into account in realistic models, namely, distribution of the satellite orbital parameters may be more complicated if two-body collisions between the orbiters are considered.

Another open question is the impact of star-disc interactions on the mass function of the satellites. We observed that $M_{*}, \Sigma_{*}$ and $\Sigma_{\mathrm{d}}$ are the crucial parameters determining the cluster evolution, but the parameter range spans an enormous interval for different types of objects. Compact bodies have $\Sigma_{*} \approx 10^{9} \Sigma_{\odot}=1.3 \times 10^{20} \mathrm{~g} \mathrm{~cm}^{-2}$ and hence they are affected less by collisions with the disc than solar-type stars, which are aligned with the disc plane more rapidly. Consequently, the initial mass function is modified towards a higher abundance of compact stars residing in inclined orbits, and vice versa. However, in the relatively dense environment of the disc, the stars accrete at an enhanced rate, they will soon gain sufficient mass and eventually collapse, producing additional compact bodies. A detailed discussion of their subsequent evolution under the influence of the disc environment remains beyond the scope of the present discussion. We only remark that solar-mass satellites can substantially multiply their masses during $10^{6}$ years (Collin \& Zahn 1999), assuming that their own radiation and the effect of gaps do not halt further accretion. This is almost two orders of magnitude shorter than the expected quasar life-time (Haehnelt \& Rees 1993) and hence the effects of star-disc interaction should not be neglected.

As a concluding remark, let us recall de nouveau that the final stages of an orbiter located near the center are relevant for gravitational wave experiments. The radiation losses govern the orbital evolution if the satellite is compact enough; on close orbits $\left(e \approx 0.9, a \approx 10^{2} r_{\mathrm{g}}\right)$ the influence of gravitational radiation is comparable to the effects of star-disc collisions, even if the medium is relatively dense, $\Sigma_{\mathrm{d}} \approx 10^{5} \mathrm{~g} \mathrm{~cm}^{-2}$.

Acknowledgements. The authors acknowledge discussions with Suzy Collin about gravitationally unstable regions of accretion discs, and useful comments and suggestions by the referee, who helped us to improve clarity of the text. Support from the grants GAUK 188/2001, GACR 205/00/1685, and 202/99/0261 is also gratefully acknowledged.

\section{Appendix A: Details on modified clusters}

We can expand the results of Sect. 3.2 in slightly more detail by examining how the modified cluster distribution depends on the adopted accretion regime and how the quasi-stationary state is eventually established. There is a qualitative difference from the simpler case discussed in the main text, because varying surface density profiles of the disc models (i)-(v) introduce potentially observable concentrations of satellites at certain radii. Also, it is instructive to recover in the graphs different influences 
(i)

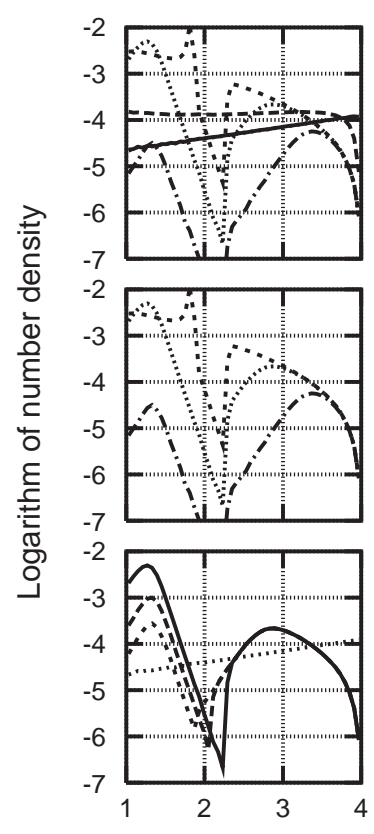

(ii)
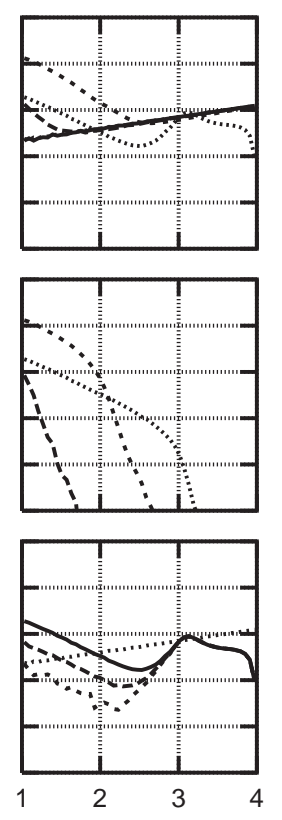

(iii)
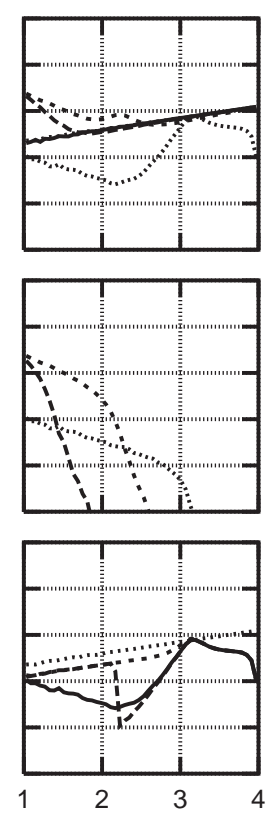

Logarithm of semi-major axis (iv)
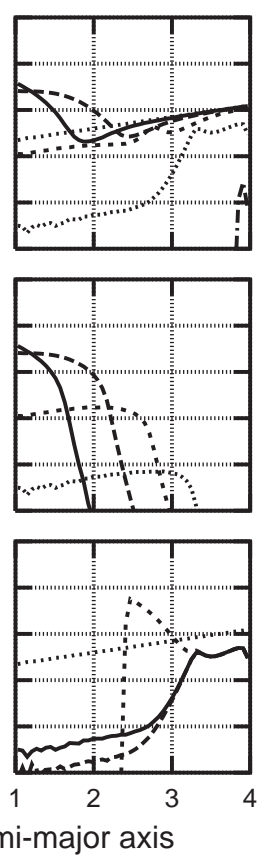

(ii)+(iii)
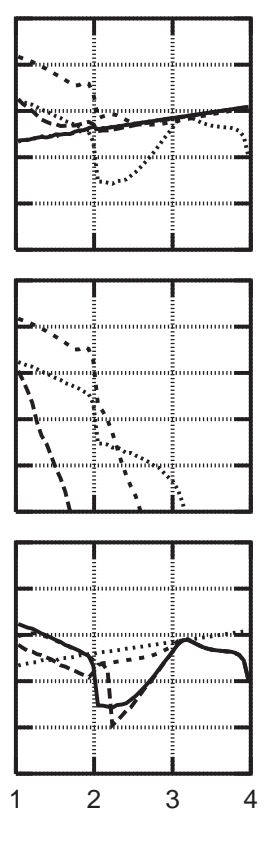

(i)+(iv)
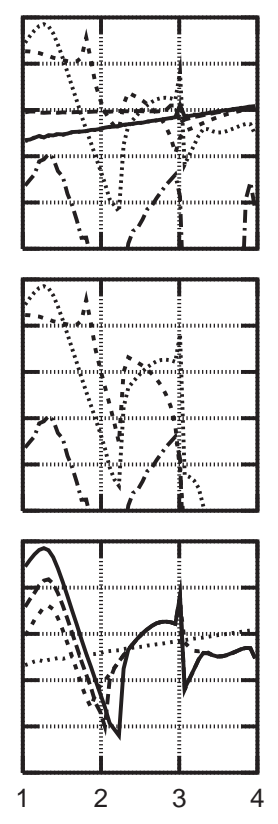

Fig. A.1. A modified distribution $n_{t}(a)$ of satellites in the sample. Columns (i)-(iv) show the modified distributions which are developed following the interaction with the corresponding disc models. (The models are listed in Sect. 3.) The last two columns reflect the structure of composite models consisting of different regions, as indicated on top. The evolution of the composite models can be compared with the case of individual simple models from which they are constructed. In each graph, line types of the curves reflect different views of $n_{t}(a)$ profiles (see the text). First, snapshots are shown with varying time (top row; $\left.M_{*}=1 M_{\odot}\right)$; second, the fraction of satellites aligned with the disc is extracted from the whole sample (middle row); third, the quasi-stationary states are plotted for different $M_{*}$ (bottom row). See the Appendix for exact interpretation of different rows of graphs.

resulting from orbital decay, discussed for individual satellites in previous paragraphs (Sect. 2).

In Fig. A.1, the first four columns correspond to simple models adopted from our list. In the remaining two columns, the disc consists of a conjunction of two different models, joined at a given radius: The model denoted (ii) + (iii) is formed by the inner (ii) and the outer (iii) part of the gas pressure dominated disc with the transition at $100 r_{\mathrm{g}}$. Similarly, the model denoted (i) $+(\mathrm{iv})$ is a conjunction of the radiation pressure dominated disc spreading up to $10^{3} r_{\mathrm{g}}$ with the outer, self-gravitating disc (Collin \& Huré 1999). The model parameters determine the final form of plotted curves in their mutual interplay together with the mass $M_{*}$ of the cluster members, which remains the characteristic influencing the efficiency of individual contributions to the cluster evolution.

Given $a$, the values of $n_{t}(a)$ are distinguished from each other if a substantial fraction of the satellites are inclined in the plane of the disc without opening a gap in it. In such a situation the migration is faster for more massive stars whose number density is then lowered with respect to low-mass stars. Radial distribution of the satellites is influenced by the mode of their migration in the disc, and as a consequence the satellites are transported at different rates towards the centre. As a result, a wiggle occurs in the $n_{t}(a)$ curve. The particular value of $a$ where the satellites are accumulated at an increased rate, forming a ring-type structure, depends on the details of the model. However, the trend to accumulation at some distance is seen in various other situations and it leads to a bias in the mass function of the cluster.

For example, when the model (ii) is considered, the stars proceed under the regime of density wave excitation in the disc plane. The critical point (below which the gap would have been opened) lies at too small distances, $\lesssim 2 r_{\mathrm{g}}$, even for the largest considered mass $M_{*}=10 M_{\odot}$. Another situation develops in the case (iii) where the gap is opened below $a=5,150$ and $7 \times 10^{3}$ for our three adopted values of satellite masses. This implies different shapes of $n_{t}(a)$ for $M_{*}=M_{\odot}$ satellites with respect to more massive ones. Furthermore, in the marginally unstable disc, i.e. the case (iv), the point above which the gap could be opened is beyond the outer boundary of the considered region and, therefore, the distributions $n_{t}(a)$ are sensitive to the mass of the satellites (most of them are inclined in the disc at this stage).

In order to maintain clarity of the graphs we omit the curves corresponding to the model $(\mathrm{v})$. This case is 
similar to (ii) except for the fact that the gap opens for all three considered stellar masses, which makes the case (v) distributions very similar to each other, quite independent of $M_{*}$.

We could see that simple power-law profiles $\Sigma_{\mathrm{d}}(r)$ lead to different distributions with respect to more realistic models, which are governed by different processes dominating at the corresponding radius. In discs that are a compound of several parts, the surface density profile contains several transitions between different regions, and hence it is more complicated than a simple power-law. The resulting effects on the boundaries resemble the transitions which we observed previously with simple discs when the mode of radial migration is changed. A local peak of $n_{t}(a)$ occurs at radii where the satellites enter the disc region with slower radial motion, which is the situation of the composite model (ii) + (iii), or quite on the contrary a dip develops in $n_{t}(a)$ as it can be seen in the case (i)+(iv).

We observed that a quasi-stationary distribution $n_{t}(a)$, different from the initial one, was established below $\lesssim 10^{3} r_{\mathrm{g}}$ for rather extended periods of time. However, a decline of $n$ eventually arrives from large $a$ at final stages when the supply of satellites is exhausted. The sample is eventually depleted. This can be also seen in Fig. A.1, the first (top) row, where time evolution is presented for one solar-mass satellites. Dimensionless times are $t=10^{6}$ (solid line), $10^{8}$ (long-dashed), $10^{10}$ (short-dashed), $10^{12}$ (dotted), and $10^{14}$ (dash-dotted). See footnote 1 for time units. The last curve is not visible in all plots because of a very large $t$; it can be noticed e.g. in the case (iv) where the evolution is slow and a fraction of satellites persists above $a \gtrsim 2 \times 10^{3}$.

Complementary to the above-described panels is the second row of graphs where only a part of $n_{t}(a)$ distribution is plotted, corresponding to the satellites aligned with the disc. Line styles denote time in the same manner as in the first row. Naturally, the population of aligned bodies is less frequent, and hence some curves are missing in the second row with respect to their matching curves in the first row, if no satellites are brought to zero inclination.

Finally, the third (bottom) row shows $n_{t}(a)$ profiles taken at $t=10^{12}$. At this moment a quasi-stationary state is already established in the form depending on the mode of satellite-disc interaction. The profiles are plotted for three different values of the satellite mass, corresponding to $M_{*}=1 M_{\odot}$ (thick solid lines), $M_{*}=3 M_{\odot}$ (longdashed lines), and $M_{*}=10 M_{\odot}$ (short-dashed lines). For example, one can check in column (ii) that the structure of the plot is ruled by the density wave regime and acquires the corresponding slope. (The initial slope $1 / 4$ is also indicated with a light dotted line.) The curves clearly reflect the fact that the orbital decay (9) depends on $M_{*}$.

\section{References}

Armitage, P. J., Zurek, W. H., \& Davies, M. B. 1996, ApJ, 470, 237

Artymowicz, P. 1994, ApJ, 423, 581

Artymowicz, P., Lin, D. N.C., \& Wampler, E. J. 1993, ApJ, 409, 592

Bahcall, J. N., \& Wolf, R. A. 1976, ApJ, 209, 214

Bekki, K. 2000, ApJ, 540, L79

Chakrabarti, S. K. 1993, ApJ, 411, 610

Collin, S., \& Huré, J.-M. 1999, A\&A, 341, 385

Collin, S., \& Zahn, J.-P. 1999, A\&A, 344, 433

Colpi, M., Mayer, L., \& Governato, F. 1999, ApJ, 525, 720

Dhurandhar, S. V., \& Vecchio, A. 2001, Phys. Rev. D, 63, 122001

Ferrarese, L., \& Merrit, D. 2000, ApJ, 539, L9

Frank, J., King, A. R., \& Raine, D. J. 1992, Accretion Power in Astrophysics (Cambridge University Press, Cambridge)

Haehnelt, M. G., \& Rees, M. J. 1993, MNRAS, 263, 168

Hughes, S. A. 2001, Phys. Rev. D, in press [gr-qc/0104041]

Ivanov, P. B., Papaloizou, J. C. B., \& Polnarev, A. G. 1999, MNRAS, 307, 79

Kato, S., Fukue, J., \& Mineshige, S. 1998, Black-Hole Accretion Disks (Kyoto University Press, Kyoto)

Kim, S. S., Morris, M., \& Lee, H. M. 1999, ApJ, 525, 228

King, A. R., \& Done, C. 1993, MNRAS, 264, 388

Lin, D. N. C., \& Papaloizou, J. C. B. 1986, ApJ, 309, 846

Nakamura, T., Oohara, K., \& Kojima, Y. 1987, Progr. Theor. Phys. Suppl., 90, 1

Narayan, R. 2000, ApJ, 536, 663

Ostriker, E. C. 1999, ApJ, 513, 252

Ostriker, J. P. 1983, ApJ, 273, 99

Peters, P. C., \& Mathews, J. 1963, Phys. Rev., 131, 435

Pineault, S., \& Landry, S. 1994, MNRAS, 267, 557

Rauch, K. P. 1995, MNRAS, 275, 628

Rauch, K.P. 1999, ApJ, 514, 725

Rees, M. J. 1998, in Black Holes and Relativistic Stars, ed. R. M. Wald (University of Chicago Press, Chicago), 79

Shlosman, I., \& Begelman, M. C. 1989, ApJ, 341, 685

Shlosman, I., \& Noguchi, M. 1993, AJ, 414, 474

Sridhar, S., \& Touma, J. 1999, MNRAS, 303, 483

Syer, D., Clarke, C. J., \& Rees, M. J. 1991, MNRAS, 250, 505

Šubr, L. 2001, Thesis (Charles University Prague), in preparation

Šubr, L., \& Karas, V. 1999, A\&A, 352, 452

Takeuchi, T., Miyama, S. M., \& Lin, D. N. C. 1996, ApJ, 460, 832

Tremaine, S. 1995, AJ, 110, 628

van der Marel, R.P., \& van den Bosch, F.C. 1998, AJ, 116, 2220

Vokrouhlický, D., \& Karas, V. 1993, MNRAS, 265, 365

Vokrouhlický, D., \& Karas, V. 1998, MNRAS, 298, 53

Ward, W. 1986, Icarus, 67, 164

Ward, W. 1997, Icarus, 126, 261

Zurek, W. H., Siemiginowska, A., \& Colgate, S. A. 1994, ApJ, 434, 46

Zwart, S. F. P., Hut, P., \& Verbunt, F. 1997, A\&A, 328, 130 